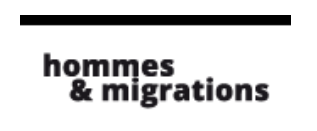

Hommes \& migrations

Revue française de référence sur les dynamiques

migratoires

1286-1287 | 2010

Les migrations subsahariennes

\title{
Le développement local en Mauritanie
}

La décentralisation à l'épreuve de la gestion de l'espace

\section{Abdoulaye Diagana}

\section{(2) OpenEdition}

1 Journals

\section{Édition électronique}

URL : http://journals.openedition.org/hommesmigrations/1759

DOI : 10.4000/hommesmigrations.1759

ISSN : 2262-3353

Éditeur

Musée national de l'histoire de l'immigration

Édition imprimée

Date de publication : 1 juillet 2010

Pagination : 246-256

ISSN : 1142-852X

Référence électronique

Abdoulaye Diagana, «Le développement local en Mauritanie », Hommes \& migrations [En ligne], 1286-1287 | 2010, mis en ligne le 29 mai 2013, consulté le 20 avril 2019. URL : http:// journals.openedition.org/hommesmigrations/1759; DOI : 10.4000/hommesmigrations.1759

Tous droits réservés 


\section{Le développement local en Mauritanie La décentralisation à l'épreuve de la gestion de l'espace}

Par Abdoulaye Diagana, doctorant en géographie, université de Rouen

Suivant son organisation administrative, la Mauritanie est subdivisée en douze régions et un district. Depuis le milieu des années quatre-vingt, répondant aux exigences internationales de bonne gouvernance, les autorités mauritaniennes ont engagé une politique de décentralisation.

Elle avait pour objectif de favoriser l'implication des citoyens dans le développement économique du pays. Cependant, le pouvoir central peine à céder de sa mainmise sur des territoires où les populations n'ont aucun droit de regard sur la gestion des représentants de l'administration. 
Les échecs successifs des différentes politiques de développement menées sous les injonctions des bailleurs de fonds extérieurs ont conduit les pays sous-développés à remettre en cause les méthodes jusqu'ici considérées comme incontournables. Cette crise du développement se révèle sous un nouveau jour avec l'ampleur prise par l'ouverture commerciale imposée par la mondialisation. La transposition dans l'espace de modèles conçus ailleurs et le défaut de rigueur dans la gestion qui a été celle des classes dirigeantes aux affaires, pour certaines, depuis plusieurs décennies ont pu peser sur le sort des politiques de développement.

Confrontés à une croissance démographique soutenue à laquelle ne correspond pas nécessairement un accroissement des ressources, les pays en mal de développement ont du également composer avec une nature

"Quarante ans plus tard, avec le recul, le discours de Truman est perçu comme le coup d'envoi de cette course du Sud pour rattraper le Nord, mais depuis, non seulement la distance s'est-elle encore agrandie et certains coureurs chancellent-ils sur la piste, mais tous commencent à soupçonner qu'ils courent peut-être tout à fait dans la mauvaise direction(1).". de plus en plus ingrate. Ce facteur combiné à la pauvreté et à la précarité qui caractérisent les populations de l'Afrique de l'Ouest a certainement eu des incidences négatives sur le sort des efforts de développement entrepris par différents acteurs.

Les nouvelles règles dictées par l'ouverture économique et commerciale à l'échelle planétaire (mondialisation) imposent aux pays africains de s'adapter à une conjoncture autrement plus exigeante que celles qu'ils ont connues jusqu'ici, notamment en réorientant leurs actions vers plus de compétitivité. Les bailleurs de fonds ont, par ailleurs, revu leur politique d'aide au développement en mettant l'accent sur la "bonne gouvernance" avec, comme pilier central, l'implication et la responsabilisation des populations, conceptrices et "maîtresses" de leur développement.

C'est ainsi que face aux carences et aux crises multiformes nées de la concentration des pouvoirs dans les centres de décision, la Mauritanie, a entrepris un transfert progressif des pouvoirs aux collectivités locales appelées à prendre en charge leur propre développement. Cette nouvelle dynamique est présentée par les pouvoirs publics comme la traduction de la volonté d'introduire de nouvelles méthodes de gestion qu'englobe le concept de "bonne gouvernance ${ }^{(2)}$ ". Il s'agit d'appréhender les caractéristiques de l'expérience mauritanienne en matière de démocratie participative qui suscite tour à tour espoirs et inquiétudes, scepticisme et interrogations ${ }^{(3)}$. 


\section{De l'autocratie à l'apprentissage du partage : le parcours erratique d'un appareill prégnant}

Introduite en Mauritanie en plusieurs étapes, la décentralisation s'est résumée le plus souvent à une décongestion administrative avec un pouvoir central contrôlant l'essentiel du jeu, loin du principal centre de décision qu'est resté Nouakchott ${ }^{(4)}$. Cette hypercentralisation se traduit par des flux démographiques vers les centres urbains et la transformation de Nouakchott, capitale d'à peine quarante ans d'existence, en un pôle qui s'oppose au reste du pays. Ce développement déséquilibré et monocéphale engendre d'importants problèmes d'infrastructures et de répartition des ressources, compte tenu de la faible capacité d'absorption d'une ville en développement constant. Pourtant, l'expérience en matière de gestion des ressources naturelles au niveau local montre que les résultats peuvent être efficients quand les populations prennent en charge une partie de leurs affaires. Pour preuve, la récurrence des discours ${ }^{(5)}$ qui militent pour l'institutionnalisation de la gestion communautaire en vue d'améliorer l'égalité et la justice et de garantir une meilleure protection de la nature sur le modèle de la Community Based Natural Reserve Management (CBNRM).

Cette option présente l'avantage de pouvoir se fonder sur l'expérience dont disposent les locaux grâce à leur proximité avec leur milieu et la parfaite connaissance qu'ils en ont. De plus, elle obéit au principe de subsidiarité qui veut que les problèmes soient résolus par l'autorité publique compétente au plus petit niveau. La répartition des attributions et des compétences est dès lors un subtil jeu d'arbitrage et de recherche d'équilibre pour mesurer le niveau le plus pertinent dans différents domaines de la vie publique, en instituant des critères définissant la bonne distance géographique, la taille critique des territoires, ainsi que les convergences et les synergies à créer ou à développer aussi bien au niveau des communes qu'à l'échelle intercommunale ou régionale.

De ce point de vue la décision des autorités mauritaniennes de "faire descendre le pouvoir vers les administrés (6)" est en soi, dans son principe, un tournant dans le mode de gestion des affaires publiques et le maillage du territoire. C'est en ce sens que l'expérience connue par le pays en $1986^{(7)}$ marque le premier pas vers une étape nouvelle dans le processus de responsabilisation des populations. Limitée dans un premier temps aux chefs-lieux de régions avec un mode de désignation semblable à celui des communes urbaines de la constitution de 1961, l'expérience est étendue dès $1987^{(8)}$ aux chefs-lieux de département puis aux communes rurales en 1988. En tout, 208 communes étaient habilitées à désigner leurs représentants, maires compris. 


\section{Un pouvoir central qui conserve sa mainmise}

Toutefois, si le principe est acquis, des doutes persistent sur la volonté des gouvernants à transférer réellement le pouvoir aux élus locaux. En effet, populations et candidats sont invités à s'exprimer uniquement durant les périodes électorales, sans possibilité de constituer des partis politiques. De plus, le gouvernement garde un droit de regard sur la municipalité en ce sens que les décisions du maire restent soumises à l'approbation préalable du ministère de tutelle (ministère de l'Intérieur, des Postes et Télécommunications) par le triptyque Approbation-Annulation-Substitution. Le système du contrôle a priori ralentit l'action des communes et limite leur autonomie. La tutelle dispose également d'un pouvoir de sanction à l'égard des autorités communales par la suspension, la dissolution ou la révocation du conseil municipal ou du maire et de ses adjoints ${ }^{(9)}$. La marge de manceuvre de l'équipe municipale est rendue d'autant plus limitée que ses ressources se résument aux seules subventions provenant de l'État qui est censé se réorganiser pour plus d'efficacité en se redéployant au niveau des collectivités territoriales.

Malgré les aménagements introduits en 1991, le nombre de communes passant à $216^{(10)}$, la situation ne changera pas fondamentalement. La nouvelle constitution adoptée en juillet 1991 introduit une nouvelle donne : la compétition se fait désormais entre partis politiques pour le suffrage des Mauritaniens, aussi bien au niveau local que national. Mais l'omnipotence du Parti Républicain Démocratique et Social, Parti-État, et la volonté du pouvoir de garder sa position de pourvoyeur de biens, de distributeur de récompenses et de prébendes finissait par installer une atmosphère peu propice aux échanges féconds, à la critique et au suivi-évaluation. Les communes qui, à l'instar de Kaédi ${ }^{(11)}$, ont fait le choix de l'opposition, ont eu à faire face à l'ostracisme de l'État. Sans illusions quant aux effets d'un processus qui n'avait manifestement d'autre objectif que de "légitimer" la mainmise de l'autorité centrale sur le pouvoir local, les populations ne l'ont accompagné que timidement.

\section{Une décentralisation en devenir}

Aujourd'hui encore, la décentralisation en Mauritanie recèle de nombreuses insuffisances et les interrogations demeurent. En dépit de l'engagement pris par les gouvernants et leurs partenaires extérieurs en vue d'une plus grande implication des populations, le transfert des compétences n'a pas été suivi d'un transfert de pouvoirs. Lordonnance 89-287 et ses textes subséquents délimitent pourtant clairement le 
découpage territorial ainsi que les niveaux d'interventions et de compétences des différentes entités. Les communes héritaient d'une quinzaine d'attributions au nombre desquelles figurent la voirie urbaine, les écoles, les centres de santé, les transports, l'hygiène, les marchés, les abattoirs, l'aide aux indigents pour mieux sacrifier au discours sacralisant la lutte contre la pauvreté.

Cependant, les critères de découpage restent flous. Des communes sont instituées à la discrétion du ministre de l'intérieur sans avoir nécessairement la viabilité économique et la masse critique d'activités nécessaires à leur développement. Les disparités en terme économique qui naissent de ce travers font qu'au final les communes ne disposent pas des mêmes outils pour affronter les défis auxquels elles font face (lutte contre la pauvreté, accès à l'eau et à la santé, protection de la nature, scolarisation...). Partant, les communes restent pour la plupart dépourvues de moyens financiers ${ }^{(12)}$ et politiques malgré la "légitimité" qu'elles tiennent du suffrage universel. De même, les élus n'ont pas les moyens de recouvrer les taxes qui pourraient constituer un important apport budgétaire en plus de la dotation du Fonds régional de développement ${ }^{(13)}$.

Il est difficile à l'observateur de ne pas avoir le sentiment que la décentralisation est adoptée comme mode de gestion non pas pour améliorer la gouvernance, mais bien pour répondre à un certain air du temps qui voudrait que l'on associe les populations juste ce qu'il faut pour se conformer aux critères des partenaires au développement. D'où les interventions récurrentes de représentants de l'État s'en prenant aux discours et prises de position hypostasiant la société civile. Cette réflexion est à rapprocher d'un sentiment largement partagé que de nombreux régimes du continent africain n'ont adhéré au mode de désignation démocratique de leurs représentants, y compris et surtout au sommet de l'État, que contraints et forcés et dans l'espoir de reprendre à couvert le pouvoir autoritaire qu'ils ont fait semblant d'abandonner à l'électeur.

\section{Entre répression et démagogie, une certaine idée de la démocratie}

Aussi, en Mauritanie nombreux sont ceux qui soutiennent que "face à la société, le pouvoir a une grande capacité à s'attribuer, sans risque d'être contesté, la paternité de linstauration du régime démocratique, quitte à en modifier la substance. Ainsi, par exemple, en illusionniste du changement, mobilise-t-il le référent démocratique en opérant de deux façons simultanées : d'abord, il installe un cadre institutionnel formellement pluraliste, mais plus ou moins opérationnel; ensuite, et comme pour désamorcer les réticences à l'égard du cadre ainsi institué, il donne l'impression de céder aux impératifs de représentativité et de 
rotation des dirigeants en opérant de fréquents changements au sein de l'équipe dirigeante." Le but est in fine de "faire participer toutes les régions, toutes les tribus, toutes les coteries, toutes les ethnies, à tour de rôle ${ }^{(14)}$ ", comme pour corriger les imperfections d'un système que l'on s'est certes résolus à importer, mais non sans quelques aménagements techniques dont le résultat se décline en une copie bien pâle et tropicalisée du modèle original. D'où provient ce paradoxe qui se traduit, d'une part, par une volonté déclarée et affichée de transférer les charges et, d'autre part, par la difficulté qu'éprouve l'État à se délester d'une partie de ses prérogatives ?

Une partie de l'explication est sans doute à rechercher dans l'absence d'une culture de gestion partagée et de délégation de l'autorité qui caractérise la relation au pouvoir en Mauritanie. Pour une population essentiellement agropastorale, la récurrence des conflits - corollaire de la raréfaction des ressources et de la compétition plus serrée pour la survie qu'elle entraîne - est l'instrument pertinent pour mesurer la lourdeur de la "main" de la puissance publique, souvent confondue avec la répression ${ }^{(15)}$. Cette situation se vérifie davantage dans la partie australe du pays, sur les rives du fleuve Sénégal. Là, la sagesse populaire enseigne que l'on ne peut ni ne doit résister à la volonté d'un représentant de l'État, y compris dans les cas flagrants d'abus. De son côté, le pouvoir, généralement acquis de façon illégitime, compense le péché originel par une démonstration excessive de puissance, à travers un appareil répressif et sécuritaire surdimensionné. Ce face-à-face pouvoir/citoyen prend un relief tout particulier dans cette partie du pays, avec la réforme foncière de 1983. 


\section{Aménagement du territoire et gestion de l'espace : quand la réalité rattrape la théorie}

Pour révolutionnaire qu'elle puisse être, la réforme foncière censée encadrer la gestion de la terre a peut être ici créé plus de torts qu'elle n'en a redressés ${ }^{(16)}$. Le contexte qui présidait à la naissance de cette réforme était bien particulier.

Attendue depuis l'accession de la Mauritanie à la souveraineté nationale ${ }^{(17)}$, cette réforme vient ordonner la gestion de l'espace caractérisée par un flou entretenu par la superposition de trois principales sources de droit : le droit d'inspiration musulmane, le droit moderne et le droit coutumier avec le mode de tenure traditionnelle du sol ${ }^{(18)}$. En disposant que "sont confirmés les droits fonciers coutumiers comportant une emprise évidente et permanente sur le sol', la loi du 2 août 1960 en son article 3 aménage aux forces traditionnelles un dispositif qui leur permettra de garder la mainmise sur la gestion de l'espace. Les seules espaces encore propriétés de l'État sont les terres mortes, celles qui sont "vacantes et sans maîtres". Il s'agit généralement de terres lointaines et difficilement exploitables, par conséquent peu porteuses d'intérêt. Ce cadre juridique laissait courir une situation caractérisée par un système dans lequel les terres utiles et les plus convoitées étaient détenues suivant des logiques privées ou communautaires déterminées selon le lignage. Le "maitre de la terre ${ }^{(19)}$ "est dès lors le chef (politique, religieux, coutumier) qui tient sa légitimé d'un héritage, d'une antériorité en terme de présence sur les lieux, de la volonté de l'État ou encore d'accords de partage entre occupants des lieux. À ce titre, il est le détenteur des droits d'exploitation et d'usage individuels ou collectifs, permanents ou temporaires, qu'il peut affecter à sa guise et dont il se sert surtout pour accentuer et perpétuer sa position dominante. Cette configuration, peu favorable à l'accession à la propriété de populations nouvellement établies, faisait par conséquent le lit de nouveaux rapports de domination/exploitation ou de l'affermage grâce auxquels des propriétaires terriens pouvaient s'assurer des droits sur une part des récoltes sans avoir à cultiver la terre ${ }^{201}$.

\section{Le poids de l'histoire}

Par ailleurs, il est à souligner que la réforme foncière suit une autre décision d'importance, à savoir l'ordonnance $n^{\circ}$ 81-234 du 9 novembre 1981 portant abolition de l'esclavage. Les populations naguère serviles pouvaient dès lors prétendre à exploiter la terre et accéder à la propriété. Mais faute d'avoir pris les mesures d'accompagnement adéquates, l'État exacerbait les tensions entre ces nouveaux 
"libres" et les détenteurs de la terre. Les mécanismes traditionnels de médiation et de règlement des conflits n'ont été mis à contribution qu'en de rares occasions. D'où l'exacerbation des haines jusqu'au sanglant conflit d'avril 1989 qui a endeuillé les terres fertiles de la Vallée dans le sillage d'un conflit frontalier avec le voisin sénégalais. La Circulaire nº 0005/MINT du 14 avril 1984 précise, par ailleurs, les raisons qui motivaient la réforme agraire : Tout citoyen doit pouvoir être titulaire d'un droit réel de propriété foncière, indépendante des propriétés collectives qui dissimulent parfois des liens de dépendance personnelle." Partant, en supprimant la propriété collective de la terre et en individualisant les droits fonciers, la réforme foncière du 5 juin $1983^{(21)}$ introduit de profondes mutations dans l'organisation sociale des communautés traditionnelles.

Sur fond de redistribution de la terre, c'est bien à une tentative de réorganisation des

Aujourd'hui, la contribution du citoyen au financement du développement local est quasi nulle. pratiques sociales que nous assistons: "L'expérience de la plaine de M'Pourié, de Bogué, de Kaédi et de Foum- Gleita, montre que le mode de tenure des terres constitue une contrainte permanente pour les aménagements hydro agricoles", poursuit la circulaire. Mais, mal préparée, cette loi donnait l'occasion aux grandes fortunes de s'engouffrer dans la brèche en faisant la promotion de l'agrobusiness sans volonté de créer une réelle dynamique de développement du secteur agricole.

Les populations locales finiront par mal accueillir l'arrivée massive d'investisseurs privés et l'application de nouvelles règles foncières, perçues comme une volonté d'expropriation et de colonisation, les agrobusinessmen étant généralement issus de la communauté araboberbère ${ }^{(22)}$ et les terres traditionnellement occupées par des populations noires. L'absence de concertation et d'implication des paysans, l'autoritarisme de l'État et le défaut de sensibilisation sont probablement à l'origine des ratés de la réforme de 1983.

\section{Répartition des champs de compétences et financements : des difficultés structurelles}

Le climat qui entourait la réforme agraire et qui la rendait inopérante parce que rejetée d'emblée, se retrouve plus ou moins en de nombreuses actions entreprises dans le cadre de la décentralisation. L'explication tient, d'une part, à la nature de la 
société (qui ne voit en l'État que la puissance publique synonyme de contrainte et de répression, donc ennemi potentiel dont les desseins sont à contrarier par tous les moyens et surtout symbole de l'entité à dépouiller autant que possible) et de l'État (centralisateur jacobin) et, d'autre part, aux carences liées à la rareté des moyens (humains et matériels). Tous ces facteurs sont de nature à annihiler les efforts fournis en matière de développement.

Administrativement coordonnée à l'origine par le ministère de l'Intérieur des Postes et Télécommunications (MINT), la politique de décentralisation vient d'échoir au tout nouveau ministère de la Décentralisation et de l'Aménagement du territoire, créé par le premier gouvernement d'après transition ${ }^{(23)}$. Jusqu'ici le choix du MINT, véritable pouvoir au cceur du pouvoir, démontrait la volonté du gouvernement de garder la haute main sur un processus qui, par essence, devait déboucher sur la responsabilisation des populations, donc à une certaine renonciation à une partie des prérogatives de l'État. La Direction générale des collectivités locales (devenue Direction générale de la gouvernance locale (DGGL)) avait hérité de la charge de mettre en ceuvre cette politique ${ }^{(24)}$, mais la faiblesse de ses moyens limitait la portée de son action. Or la DGGL est à ce jour le principal organe technique chargé de la conception et de la mise en ceuvre de la politique de gestion partagée du pouvoir.

Pour rapprocher le citoyen du pouvoir sur un très vaste territoire occupé de façon disparate par une population qui n'est pas encore complètement sédentarisée, l'État mauritanien a mis en place un Fonds régional de développement dont le budget est passé de 265 millions en 2001 à 2 milliards 500 millions d'ouguiyas ${ }^{(2)}$ en 2006. Pour l'année 2007, il était prévu de lui affecter un pourcentage du budget national ${ }^{(26)}$. Cette affectation est à ce jour la principale source de revenus pour de nombreuses communes. Le fonds est subdivisé en trois dotations: la dotation au fonctionnement, qui représente $35 \%$ du FRD, dont $15 \%$ à parts égales entre les communes et le reste réparti en fonction de la taille démographique et du taux de pauvreté de chaque wilaya ${ }^{(27)}$; la dotation d'équipement, qui couvre $55 \%$ du FRD, dont $40 \%$ affectés en fonction du poids démographique de la commune et $15 \%$ représentant la part compensatrice du retard d'équipement et une dotation pour le suivi évaluation et le renforcement des capacités de l'Administration. Gérée à l'origine par le ministère de l'Intérieur, elle vient d'échoir au ministère de la Décentralisation et de l'Aménagement du territoire.

Mais ici, comme au sujet de l'expérimentation de concepts transférés, le sentiment est largement partagé que l'État et certains acteurs du développement anticipent et s'approprient la notion de gestion partagée, le plus souvent afin de répondre aux critères fixés par les bailleurs extérieurs et de bénéficier ainsi de la manne des subventions. Des élus et des agents de développement se plaignent de la gestion 
catastrophique de ces fonds dont l'intégralité n'arriverait pas à destination. À travers ces quelques éléments, c'est toute la politique du contrôle du territoire national qui gagnerait à être clarifiée.

\section{Conclusion}

À se pencher sur le cadre légal et les orientations de la Déclaration de politique municipale ${ }^{(28)}$, force est de constater que si les autorités s'engagent à rapprocher l'administration du citoyen en assurant une couverture efficiente du territoire national, les modalités de financement de cette politique laissent apparaître quelques faiblesses. À l'inverse de l'expérience malienne ${ }^{(29)}$, la réflexion n'est pas allée jusqu'à remettre en cause le découpage actuel aux fins de créer des entités qui soient économiquement viables. Responsabiliser les communes en matière fiscale aurait eu deux effets. Le premier étant d'impliquer le citoyen et d'inciter à une meilleure gestion pour de meilleurs résultats. Le second étant de développer la notion de chose publique et le sentiment d'appartenance à une entité commune partageant le même destin.

La position de l'État n'en est pas moins paradoxale: tout en tenant à assurer un contrôle total du processus de développement local au niveau national (centralisation) et en prétendant vouloir associer le citoyen à la définition et la conduite de ses propres affaires, l'État "oublie" de demander à celui-ci de lui témoigner davantage sa fidélité et son allégeance en contribuant au financement de cette politique à travers le paiement de l'impôt. Aujourd'hui, la contribution du citoyen au financement du développement local est quasi nulle. Les rares ressources qui pourraient provenir d'une collecte locale sont les taxes qui, au nom d'une certaine forme de "privatisation" de la chose publique restent le trésor de guerre de l'agent collecteur ${ }^{(30)}$.

Pourtant, l'État n'aurait pas eu à renoncer à une importante fortune en "confiant" la mission de collecte aux collectivités locales : même dans les grands centres urbains (Nouakchott et Nouadhibou compris) la collecte de l'impôt est insignifiante en dehors de la part d'éventuelles entreprises présentes dans leur périmètre. Parallèlement à cette délégation de pouvoir, l'État pourrait également exiger une meilleure implication de la société civile pour assurer une certaine transparence et un contrôle démocratique de la gestion au niveau régional ou local. Le but ultime étant de laisser les communes définir elles-mêmes, par elles et pour elles, les stratégies les conduisant à financer leur propre développement dans des secteurs à définir avec le pouvoir central, y compris en recourant à des financements privés, le tout dans une transparence dont le citoyen serait le garant en contrepartie de sa mobilisation. 
1. Sachs, W., Les ruines du développement, Montréal, Canada, Ecosociété, 1996, p 14.

2. Anonyme, Rapport du Comité interministériel chargé de la bonne gouvernance, République islamique de Mauritanie, Présidence de la République, octobre 2005.

3. Cet article a été rédigé avant le coup d'État militaire du 6 août 2008 qui a entrainé des modifications substantielles dans l'armature administrative de la décentralisation en Mauritanie : le ministère de la Décentralisation et de l'Aménagement du territoire a rejoint le giron du ministère de l'Intérieur. De plus, les partenaires au développement ont suspendu leur coopération avec la Mauritanie, qui vient juste de reprendre. La table ronde de Bruxelles (22, 23 juin 2010) s'est soldée par près de 3 milliards de dollars US de promesses de dons. Il s'agit pour l'essentiel de promesses faites lors de la table ronde de Paris (2007) et qui n'avaient pas été concrétisées suite à la rupture de l'ordre constitutionnelle survenue en 2008.

4. La première expérience en matière de décentralisation peut être située aux lendemains de l'indépendance. La constitution de 1961 prévoyait, en effet, trois types de communes : des communes urbaines qui disposaient d'un conseil élu dont l'un des membres était désigné maire ; des communes pilotes qui avaient le pouvoir d'élire leur conseil mais dirigé par un délégué du gouvernement ; les communes rurales qui pouvaient élire leur conseil tout en ayant le chef de subdivision pour faire office de maire. En 1968, cette expérience est abandonnée pour céder la place à une nouvelle organisation territoriale se traduisant par la création de 8 puis 12 régions en plus du district de Nouakchott. Les conseillers et l'exécutif régional sont désignés par le gouvernement.

5. Ribot, J-C., La décentralisation démocratique des ressources naturelles, institutionnaliser la participation populaire, Washington, Institut des ressources mondiales, 2002.

6. Il s'agit-là de la traduction littérale de la décentralisation par certaines populations locales notamment les soninkés. "Hanka yankhanté" voulant dire pouvoir transféré au niveau inferieur.

7. Anonyme, Ordonnance 86-134 du 13 août 1986, abrogée par l'ordonnance 87-289 du 20 octobre 1987 (cf. note 8).

8. Anonyme, Ordonnance 87-289 du 20 octobre 1987, in Journal Officiel de la République islamique de Mauritanie, $\mathrm{n}^{\circ}$ 696-697, date de publication : 28.10.1987, pp. 403-413.

9. À l'instar de Mohamed Mahmoud Ould Mah, maire de Nouakchott démis de ses fonctions au milieu des années quatre-vingt.

10. En 2001, le décret 2001-070 du 28 juin 2001 crée 9 nouvelles communes en lieu et place de celle du district. Ce qui porte le nombre de communes à 216.

11. Principale ville du Sud de la Mauritanie, capitale de la région du Gorgol sur les rives du fleuve Sénégal.

12. La fiscalité communale est régie par l'Ordonnance 90-04 du 6 février 1990 et ses textes modificatifs (Lois de finances 1994, 2001 et 2002).

13. Pourtant l'article 89 de l'ordonnance $87-289$ dispose qu'une "convention déterminant les biens et les services transférés à la commune est conclue entre les autorités et le maire". Cependant, en dehors de cas rarissimes tels Nouadhibou et Nouakchott, cette disposition n'a pas été suivie d'effet.

14. Ould Ahmed Salem, Z., "La démocratie en Mauritanie, une 'illusion' postcoloniale", in Politique Africaine, $\mathrm{n}^{\circ} 75$, Paris, Karthala, octobre 1999, pp 131-146.

15. En langue soninké, l'État se traduit par "Hanka", qui signifie la force. D'où Hanka heti gajalemma (L'État n'est pas un adversaire), dans le sens où les forces étant disproportionnées, l'individu ne peut que perdre dans la confrontation. Il s'agit plus d'une attitude dictée par le réalisme que par le désir de faire preuve d'un sens civique irréprochable.

16. Schmitz, J., "Anthropologie des conflits fonciers et hydropolitiques du fleuve Sénégal (1975-1991)", in Cahiers des Sciences Humaines, vol. 29, n ${ }^{4}$, ORSTOM, 1993, pp. 591-623.

17. Hesseling G. et Crousse B., La réforme foncière dans la vallée du fleuve Sénégal en Mauritanie. Son impact sur les populations locales, Rapport de mission pour la Banque Mondiale, Leiden, octobre 1992, 59 p.

18. Crousse B., "Étatisation ou individualisation, la réforme foncière de 1983 ", in Politique Africaine, $n^{\circ} 21$, mars 1986 , pp 63-76.

19. Boutillier, J-L., Schmitz, J., "Gestion traditionnelle des terres (système de décrue/système pluvial) et transition vers l'irrigation. Le cas de la vallée du Sénégal", in Cahiers des Sciences Humaines, vol. 23, "Systèmes de production agricole en Afrique Tropicale : Système de production et de développement", ORSTOM, 1987, pp. 533-554.) 
20. En pular, langue de l'ethnie des Haalpular qui occupent en partie le Sud de la Mauritanie, cette pratique de l'affermage se traduit par le rem peccen : "Tu cultives ma terre, nous partageons la récolte."

21. Ordonnance $n^{\circ} 83-127$, in Journal Officiel de la République islamique de Mauritanie, $n^{\circ} 592-593$, date de promulgation : 05.06.1983, date de publication : 29.06.1983, p.364.

22. La part de responsabilité de ces changements et de la perception qu'ils engendrèrent n'est pas négligeable dans la naissance du conflit frontalier entre le Sénégal et la Mauritanie, qui contrairement à ce qui est souvent avancé n'est pas le fruit d'un conflit entre éleveurs mauritaniens et agriculteurs sénégalais. L'incident de Diawara ne fut guère que la cause immédiate d'un conflit qui sourdait de longue date et qui est le fruit d'une accumulation de frustrations qu'aggrave la trop forte pression sur les ressources en raréfaction.

23. Cf. avertissement en introduction de ce texte.

24. Décret 90-94 du 23 octobre 1994. Aujourd'hui la DGCL est rattachée au ministère de la Décentralisation et de l'Aménagement du territoire. Voir Lods, J.-L., Le financement de la décentralisation en République islamique de Mauritanie, Nouakchott, DGCL-SCAC, 2006.

25. La monnaie nationale, 1 euros équivalait à 340 ouguiyas en 2006.

26. Source ONS. À notre connaissance cette mesure n'a pas encore vu le jour.

27. La wilaya correspond à la région dirigée par un représentant nommé par le gouvernement, la décentralisation n'ayant pas atteint l'échelon régional. Le pays en compte 13 en plus du district de Nouakchott.

28. Anonyme, Déclaration de politique municipale, 1995, 2007, République islamique de Mauritanie, ministère de la Décentralisation et de l'Aménagement du territoire.

29. Gonin P., Kotlok N. et Lima S., "Entre réseaux et territoires, des mobilisations multiscalaires pour le développement. Réseaux migratoires et communes rurales dans la région de Kayes, Mali", Communication au colloque de l'université de Rennes, CRAPE et ESO : Espace de vie, espaces-enjeux, entre investissements ordinaires et mobilisations politiques", novembre 2008, pp. 5-7.

http://eso.cnrs.fr/TELECHARGEMENTS/colloques/rennes_11_08/Gonin_Patrick_Kotlok_Nathalie_Lima_Stephanie.pdf

30. Mbembé A., "Du gouvernement privé indirect", in Politique africaine, ${ }^{\circ}$ 73, Paris, Karthala, 1999, pp 103-121. 